\title{
On the Structure of Multiple Translational Tilings by Polygonal Regions*
}

\author{
M. N. Kolountzakis \\ Department of Mathematics, University of Crete, \\ Knossos Avenue, 71409 Iraklio, Greece \\ kolount@math.uch.gr
}

\begin{abstract}
We consider polygons with the following "pairing property": for each edge of the polygon there is precisely one other edge parallel to it. We study the problem of when such a polygon $K$ tiles multiply the plane when translated at the locations $\Lambda$, where $\Lambda$ is a multiset in the plane. The pairing property of $K$ makes this question particularly amenable to Fourier analysis. As a first application of our approach we establish a necessary and sufficient condition for $K$ to tile with a given lattice $\Lambda$. (This was first found by Bolle for the case of convex polygons-notice that all convex polygons that tile, necessarily have the pairing property and, therefore, our theorems apply to them.) Our main result is a proof that a large class of such polygons tile multiply only quasi-periodically, which for us means that $\Lambda$ must be a finite union of translated two-dimensional lattices in the plane. For the particular case of convex polygons we show that all convex polygons which are not parallelograms tile multiply only quasi-periodically, if at all.
\end{abstract}

\section{Introduction}

In this paper we study multiple tilings of the plane by translates of a polygonal region of a certain type, the polygons with the pairing property of Definition 2 below.

Definition 1 (Tiling). Let $K$ be a measurable subset of $\mathbb{R}^{2}$ and let $\Lambda \in \mathbb{R}^{2}$ be a discrete multiset (i.e., its underlying set is discrete and each point has finite multiplicity). We say

\footnotetext{
* This research was partially supported by the U.S. National Science Foundation, under Grant DMS 97-05775. Most of this work was carried out while the author was visiting the University of Illinois at UrbanaChampaign in Fall 1998-99.
} 
that $K+\Lambda$ is a (translational, multiple) tiling of $\mathbb{R}^{2}$ if

$$
\sum_{\lambda \in \Lambda} \mathbf{1}_{K}(x-\lambda)=w
$$

for almost all (Lebesgue) $x \in \mathbb{R}^{2}$, where the weight or level $w$ is a positive integer and $\mathbf{1}_{K}$ is the indicator function of $K$.

Whenever we speak of a tiling in this paper we mean a multiple tiling at some integer level.

Before defining the polygons with the "pairing property" we make some intuitively obvious remarks: If $K$ is a polygonal region (not necessarily connected), which tiles the plane, then each edge $e$ of a translate of $K$ which participates in the tiling must be "countered" by other edges which come from other copies of $K$. These edges must have the same direction as $e$ and at least one of them must have its outward-pointing normal vector oriented opposite to that of $e$. Hence, for a polygonal region to have any hope to tile by translation the following must hold: for any edge e of $K$ there is at least another one with the same direction whose outward-pointing normal vector is opposite to that of $e$. The situation becomes much simpler if that other edge is uniquely defined.

Definition 2 (Polygons with the Pairing Property). A polygon $K$ has the pairing property if for each edge $e$ there is precisely one other edge of $K$ parallel to $e$ and this edge has the same length as $e$.

Remarks. 1. Note that all symmetric convex polygons have the pairing property and it is not hard to see that all convex polygons that tile by translation are necessarily symmetric.

2. The polygonal regions we deal with are not assumed to be connected.

3 . The requirement that for every edge in the polygon the other edge of the same direction has the same length is not necessary, in the sense that, if a polygon admits multiple tilings by translations, it is always true, assuming only the uniqueness of the other edge of the same direction.

Using Fourier analysis we prove that a large class of polygons with the pairing property can only tile in a "quasi-periodic" manner, if they tile at all.

Definition 3 (Quasi-Periodic Multisets). A multiset $\Lambda \subseteq \mathbb{R}^{d}$ is called quasi-periodic if it is the union of finitely many $d$-dimensional lattices (see Definition 6) in $\mathbb{R}^{d}$.

Dealing with multiple tilings of space often brings in added difficulty and quite different behavior from simple (level 1) tilings by, say, polyhedra, and this is true even if one deals with lattice tilings only. As an example, we mention Minkowski's conjecture (a theorem of Hajós since 1940) which states that in every lattice tiling (at level 1) of Euclidean space by translates of the unit cube at least two cubes share an entire face of codimension one. However, this ceases to be true for multiple lattice tilings by the unit cube, when the dimension is larger than three (see, for example, [SS]). 
Also, although the convex polygons that tile by translation at level 1 have long been known to be just the parallelograms and the symmetric hexagons, there are many more polygons that tile multiply, as Bolle's theorem (see Section 2) states. This implies that there are multiple tilings by polygons which are indecomposable, that is, they cannot be made up by overlapping simpler tilings and, therefore, the study of multiple tilings encompasses more than just simple tilings. One can see a concrete example of such an indecomposable multiple tiling, at level 239, by a nonregular octagon on p. 648 of [GS].

This paper is organized as follows:

In Section 1 we describe the general approach to translational tiling using the Fourier Transforms of certain measures which are supported on pairs of opposite edges and, in particular, their zero-set. These zero-sets are then calculated explicitly for polygons with the pairing property.

As a first application of our Fourier analytic method, and a demonstration that, using it, some problems become almost a matter of calculation, we give in Section 2 a necessary and sufficient condition (Theorem 2 ) for a polygon $K$ with the pairing property to tile multiply with a lattice $\Lambda$. This has been proved (by elementary methods) previously by Bolle for the more special case of convex polygons (although his method probably applies for the case of pairing polygons as well). Our approach is based on the calculation of Section 1. Another case when the Fourier analytic method has proved to be very useful can be found in [K2] where the author, after explicitly calculating the Fourier Transform of the indicator function of certain polyhedra, was able to demonstrate the existence of a lattice of the appropriate volume in their zero-set, thus (in a manner very similar to that described in Section 1) establishing the fact that these polyhedra are lattice tiles.

The main contribution of this paper comes in Section 3. There we find a very large class of polygons with the pairing property that tile only in a quasi-periodic manner. In particular we show that every convex polygon that is not a parallelogram can tile (multiply) only in a quasi-periodic way (meaning translated at a finite union of lattices), if it can tile at all. This result should be viewed in the context of the so-called Periodic Tiling Conjecture (see, e.g., [KL]) which states that any domain that tiles (at level 1) $\mathbb{R}^{d}$ by translation can also tile space in a periodic manner (this means that the tiling has a $d$-dimensional period lattice).

Here is an outline of our approach using the Fourier Transform. The tiling condition, that $K+\Lambda$ is an $m$-fold tiling of $\mathbb{R}^{2}$, implies that (and, under additional assumptions, is equivalent to the fact that) the Fourier Transform of $\sum_{\lambda \in \Lambda} \delta_{\lambda}$ (a unit mass at each point of $\Lambda$ ) is supported on the zero-set of the Fourier Transform of the measure $\mu_{e}$, where $\mu_{e}$ is the measure which charges with its arc-length the edge $e$ of the polygon and with its negative arc-length the edge opposite to $e$. This support property should hold for every $e$. For most polygons with the pairing property this intersection (over all different edges $e$ ) of the zero-sets turns out to be discrete. We then use a theorem of Meyer which greatly restricts the point-sets $\Lambda$ which give rise to such a Fourier Transform being supported on a discrete set. The structure for $\Lambda$ that comes out of Meyer's theorem is a product of the so-called "idempotent theorem" of Cohen for general locally compact abelian groups. The work is completed by a careful study of what Meyer's theorem actually gives in this case. That is, we study the structure of the "discrete coset ring" of $\mathbb{R}^{2}$ (see Section 3 for a definition). 
Notation. 1. The Fourier Transform of a function $f \in L^{1}\left(\mathbb{R}^{d}\right)$ is normalized as follows:

$$
\widehat{f}(\xi)=\int_{\mathbb{R}^{d}} e^{-2 \pi i\langle\xi, x\rangle} f(x) d x
$$

2. The action of a tempered distribution (see [R2]) $\alpha$ on a function $\varphi$ of Schwarz class is denoted by $\alpha(\varphi)$. The Fourier Transform $\widehat{\alpha}$ of $\alpha$ is a tempered distribution defined by

$$
\widehat{\alpha}(\varphi)=\alpha(\widehat{\varphi}) .
$$

A tempered distribution $\alpha$ is supported on a closed set $K$ if for each smooth function $\varphi$ with $\operatorname{supp} \varphi \subset K^{c}$ we have $\alpha(\varphi)=0$. The intersection of all such closed sets $K$ is called the support of $\alpha$ and denoted by $\operatorname{supp} \alpha$.

\section{The Fourier Analytic Approach}

\subsection{General}

Suppose $K$ is a polygon with the pairing property and let $e_{1}$ and $e_{2}$ be two edges of the same direction $u$ and the same length. We can then write (here $e_{1}$ and $e_{2}$ are viewed as point-sets in $\mathbb{R}^{2}$ and $\tau$ as a vector)

$$
e_{2}=e_{1}+\tau
$$

for some $\tau \in \mathbb{R}^{2}$. (For each set $A$ and vector $x$ we write $A+x=\{a+x: a \in A\}$.) Then let $\mu_{u}$ be the measure which is equal to the arc-length on $e_{1}$ and the negative arc-length on $e_{2}$. Suppose also that $K+\Lambda$ is a multiple tiling of $\mathbb{R}^{2}$. Since every part of a translate of $e_{1}$ in the tiling has to be canceled by part of a copy of $e_{2}$ it follows that

$$
\sum_{\lambda \in \Lambda} \mu_{u}(x-\lambda)
$$

is the zero measure in $\mathbb{R}^{2}$. It is also intuitively obvious that the vanishing of the above measure for all directions $u$ also implies tiling at some integer level.

So a polygon $K$ with the pairing property tiles multiply with a multiset $\Lambda$ if and only if, for each pair $e$ and $e+\tau$ of parallel edges of $K$,

$$
\sum_{\lambda \in \Lambda} \mu_{e}(x-\lambda)=0
$$

where $\mu_{e}$ is the measure in $\mathbb{R}^{2}$ that is the arc-length on $e$ and the negative arc-length on $e+\tau$. Write

$$
\delta_{\Lambda}=\sum_{\lambda \in \Lambda} \delta_{\lambda}
$$

where $\delta_{a}$ is a unit point mass at $a$. Thus $\delta_{\Lambda}$ is locally a measure but is globally unbounded when $\Lambda$ is infinite. However, whenever $K+\Lambda$ is a multiple tiling, it is obvious that $\Lambda$ cannot have more than $c R^{2}$ points in any disk of radius $R, R>1$ ( $c$ depends on $K$ and 
the weight of the tiling). This implies that $\delta_{\Lambda}$ is a tempered distribution and we can take its Fourier Transform, denoted by $\widehat{\delta_{\Lambda}}$. Condition (1) then becomes $\mu_{e} * \delta_{\Lambda}=0$ or, taking Fourier Transforms,

$$
\widehat{\mu_{e}} \cdot \widehat{\delta_{\Lambda}}=0
$$

When $\Lambda$ is a lattice $\Lambda=A \mathbb{Z}^{2}$, where $A$ is a $2 \times 2$ invertible matrix, its dual lattice $\Lambda^{*}$ is defined by

$$
\Lambda^{*}=\left\{x \in \mathbb{R}^{2}:\langle x, \lambda\rangle \in \mathbb{Z}, \forall \lambda \in \Lambda\right\},
$$

and we have $\Lambda^{*}=A^{-\top} \mathbb{Z}^{2}$. The Poisson Summation Formula then takes the form

$$
\widehat{\delta_{\Lambda}}=\operatorname{det} \Lambda \cdot \delta_{\Lambda^{*}}
$$

Since $\widehat{\mu_{e}}$ is a continuous function we have in this case, and whenever $\widehat{\delta_{\Lambda}}$ is locally a measure, that condition (2) is equivalent to

$$
\operatorname{supp} \widehat{\delta_{\Lambda}} \subseteq Z\left(\widehat{\mu_{e}}\right),
$$

where for every continuous function $f$ we write $Z(f)$ for the set of points where it vanishes. (If we do not know that $\widehat{\delta_{\Lambda}}$ is locally a measure we cannot infer tiling given (4). That is, (4) does not imply (2), simply because for a function to "kill" a tempered distribution it must vanish to high enough order. For example, $x \cdot \delta_{0}^{\prime}$ is a nonzero distribution even though the support of $\delta_{0}^{\prime}$ (the derivative of the unit point mass at zero) is just the point 0 where $x$ vanishes.) When $\Lambda$ is a lattice (2) is equivalent to

$$
\widehat{\mu_{e}}(x)=0, \quad \forall x \in \Lambda^{*} .
$$

So, to check if a given polygon $K$ with the pairing property tiles multiply $\mathbb{R}^{2}$ with the lattice $\Lambda$, one has to check that $\widehat{\mu_{e}}$ vanishes on $\Lambda^{*}$ for every edge $e$ of $K$.

\subsection{The Shape of the Zero-Set}

Here we study the zero-set of the Fourier Transform of the measure $\mu_{e}$ of Section 1.1 and determine its structure. The result (Theorem 1) will be used throughout the rest of the paper.

We first calculate the Fourier Transform of $\mu_{e}$ in the particular case when $e$ is parallel to the $x$-axis, for simplicity. Let $\mu \in M\left(\mathbb{R}^{2}\right)$ be the measure defined by duality by

$$
\mu(\varphi)=\int_{-1 / 2}^{1 / 2} \varphi(x, 0) d x, \quad \forall \varphi \in C\left(\mathbb{R}^{2}\right) .
$$

That is, $\mu$ is the arc-length on the line segment joining the points $\left(-\frac{1}{2}, 0\right)$ and $\left(\frac{1}{2}, 0\right)$. Calculation gives

$$
\widehat{\mu}(\xi, \eta)=\frac{\sin \pi \xi}{\pi \xi} .
$$

Notice that $\widehat{\mu}(\xi, \eta)=0$ is equivalent to $\xi \in \mathbb{Z} \backslash\{0\}$. 
If $\mu_{L}$ is the arc-length measure on the line segment joining $(-L / 2,0)$ and $(L / 2,0)$ we have

$$
\widehat{\mu_{L}}(\xi, \eta)=\frac{\sin \pi L \xi}{\pi \xi}
$$

and

$$
Z\left(\widehat{\mu_{L}}\right)=\left\{(\xi, \eta): \xi \in L^{-1} \mathbb{Z} \backslash\{0\}\right\} .
$$

Write $\tau=(a, b)$ and let $\mu_{L, \tau}$ be the measure which is the arc-length on the segment joining $(-L / 2,0)$ and $(L / 2,0)$ translated by $\tau / 2$ and the negative arc-length on the same segment translated by $-\tau / 2$. That is, we have

$$
\mu_{L, \tau}=\mu_{L} *\left(\delta_{\tau / 2}-\delta_{-\tau / 2}\right)
$$

and, taking Fourier Transforms, we get

$$
\widehat{\mu_{L, \tau}}(\xi, \eta)=-2 \frac{\sin \pi L \xi}{\pi \xi} \sin \pi(a \xi+b \eta) .
$$

Define $u=\tau /|\tau|^{2}$ and $v=(1 / L, 0)$. It follows that $\left(u^{\perp}\right.$ is a unit vector orthogonal to $\left.u\right)$

$$
Z\left(\widehat{\mu_{L, \tau}}\right)=\left(\mathbb{Z} u+\mathbb{R} u^{\perp}\right) \cup\left(\mathbb{Z} \backslash\{0\} v+\mathbb{R} v^{\perp}\right) .
$$

(Each of the two summands in the union above corresponds to each of the factors in the formula for $\widehat{\mu_{L, \tau}}$.) This is a set of straight lines of direction $u^{\perp}$ spaced by $|u|$ and containing zero plus a similar set of lines of direction $v^{\perp}$ spaced by $|v|$ and containing zero. However in the latter set of parallel lines the straight line through zero has been removed. We state this as a theorem for later use, formulated in a coordinate-free way.

Definition 4 (Geometric Inverse of a Vector). The geometric inverse of a nonzero vector $u \in \mathbb{R}^{2}$ is the vector

$$
u^{*}=\frac{u}{|u|^{2}}
$$

Theorem 1. Let e and $e+\tau$ be two parallel line segments (translated by $\tau$, of magnitude and direction described by e, symmetric with respect to zero). Let also $\mu_{e, \tau}$ be the measure which charges $e$ with its arc-length and $e+\tau$ with its negative arc-length. Then

$$
Z\left(\widehat{\mu_{e, \tau}}\right)=\left(\mathbb{Z} \tau^{*}+\mathbb{R} \tau^{* \perp}\right) \cup\left(\mathbb{Z} \backslash\{0\} e^{*}+\mathbb{R} e^{* \perp}\right) .
$$

\section{When Does a Polygon Tile with a Certain Lattice?}

The following theorem has been proved by Bolle [B] who used combinatorial methods.

Theorem (Bolle). A convex polygon $K$, which is centrally symmetric about zero, tiles multiply with the lattice $\Lambda$ (for some weight $w \in \mathbb{N}$ ) if and only if for each edge ef $K$ 
the following two conditions are satisfied:

(i) In the relative interior of e there is a point of $\frac{1}{2} \Lambda$.

(ii) If the midpoint of $e$ is not in $\frac{1}{2} \Lambda$, then the vector $e$ is in $\Lambda$.

Remark. Notice that Bolle's theorem implies that all centrally symmetic convex polygons with vertices in $\Lambda$ tile multiply with $\Lambda$ at some level.

We prove the following which is easily seen to be a generalization of Bolle's theorem to polygons with the pairing property.

Theorem 2. If the polygon $K$ has the pairing property and $\Lambda$ is a lattice in $\mathbb{R}^{2}$, then $K+\Lambda$ is a multiple tiling of $\mathbb{R}^{2}$ if and only if, for each pair of edges $e$ and $e+\tau$ of $K$,

(i) $\tau \in \Lambda$, or

(ii) $e \in \Lambda$ and $\tau+\theta e \in \Lambda$, for some $0<\theta<1$.

Proof of Theorem 2. Once again we simplify matters and take the edge $e$ to be parallel to the $x$-axis and follow the notation of Section 1.1.

For an arbitrary nonzero vector $w \in \mathbb{R}^{2}$ define the group

$$
G(w)=\mathbb{Z} w+\mathbb{R} w^{\perp},
$$

which is a set of straight lines in $\mathbb{R}^{2}$ of direction $w^{\perp}$ spaced regularly at distance $|w|$. It follows that

$$
Z\left(\widehat{\mu_{L, \tau}}\right) \subseteq G(u) \cup G(v),
$$

where $u=\tau^{*}$ and $v=e^{*}$. From Theorem 1 it follows that $\Lambda^{*} \subseteq Z\left(\widehat{\mu_{L, \tau}}\right)$ which implies that $\Lambda^{*} \subset G(u)$ or $\Lambda^{*} \subset G(v)$.

This is a consequence of the following.

Observation 1. If $G, H, K$ are groups and $G \subseteq H \cup K$, then $G \subseteq H$ or $G \subseteq K$.

For, if $a \in G \backslash K$ and $b \in G \backslash H$, then $a \cdot b \in H$, say, which implies $b \in H$, a contradiction.

So we have the two alternatives

1. $\Lambda^{*} \subset G(u)$, and

2. $\Lambda^{*} \subset G(v)$.

However, since not all of $G(v)$ is in $Z\left(\widehat{\mu_{L, \tau}}\right)$, if alternative 2 holds and alternative 1 does not, it follows that

$$
\Lambda^{*} \subseteq \operatorname{span}_{\mathbb{Z}}\{v, w\},
$$

where $w$ is the smallest (in length) multiple of $v^{\perp}$ which is in $G(u)$, i.e.,

$$
w=\left(0, \frac{1}{b}\right) .
$$


We have that (6) is equivalent to

$$
\Lambda \supseteq\left(\operatorname{span}_{\mathbb{Z}}\{v, w\}\right)^{*}=\mathbb{Z}(L, 0)+\mathbb{Z}(0, b),
$$

which is in turn equivalent to

$$
(L, 0) \in \Lambda \quad \text { and } \quad(0, b) \in \Lambda
$$

Notice also that

$$
\Lambda^{*} \subseteq G(u) \quad \Longleftrightarrow \quad \Lambda \supseteq G(u)^{*} \Longleftrightarrow \Lambda \ni \frac{u}{|u|^{2}}=\tau .
$$

We have therefore proved the following lemma.

Lemma 1. If $\Lambda$ is a lattice, $u=(a, b) /\left(a^{2}+b^{2}\right)$, and $v=(L, 0)$, then

$$
\Lambda^{*} \subset\left(\mathbb{Z} u+\mathbb{R} u^{\perp}\right) \cup\left(\mathbb{Z} \backslash\{0\} v+\mathbb{R} v^{\perp}\right)
$$

if and only if

1. $(a, b) \in \Lambda$, or

2. $(L, 0) \in \Lambda$ and $(0, b) \in \Lambda$.

Allowing for a general linear transformation, let $\tau, e \in \mathbb{R}^{2}$, and let $\mu_{e, \tau}$ be the measure that "charges" with its arc-length the line segment $e$ translated so that its midpoint is at $\tau / 2$ and charges with its negative arc-length the line segment $e$ with its midpoint at $-\tau / 2$. We have proved the following:

$$
\Lambda^{*} \subset Z\left(\widehat{\mu_{e, \tau}}\right) \Longleftrightarrow\left\{\begin{array}{ll}
\tau \in \Lambda, & \text { or } \\
e \in \Lambda & \text { and } \tau+\theta e \in \Lambda,
\end{array} \text { for some } 0<\theta<1 .\right.
$$

This completes the proof of Theorem 2.

\section{Polygons that Tile Only Quasi-Periodically}

\subsection{Meyer's Theorem}

We now deal with the following question: which polygons with the pairing property admit only quasi-periodic multiple tilings. The main tool here, as it was in [KL], is the idempotent theorem of Cohen for general locally compact abelian groups, in the form of the following theorem of Meyer [M].

Definition 5 (The Coset Ring). The coset ring of an abelian group $G$ is the smallest collection of subsets of $G$ which is closed under finite unions, finite intersections, and complements (that is, the smallest ring of subsets of $G$ ) and which contains all cosets of $G$. 
Remark. When the group is equipped with a topology one usually only demands that the open cosets of $G$ are in the coset ring, but we take all cosets in our definition.

Theorem (Meyer). Let $\Lambda \subseteq \mathbb{R}^{d}$ be a discrete set and let $\delta_{\Lambda}$ be the Radon measure

$$
\delta_{\Lambda}=\sum_{\lambda \in \Lambda} c_{\lambda} \delta_{\lambda}, \quad c_{\lambda} \in S
$$

where $S \subseteq \mathbb{C} \backslash\{0\}$ is a finite set. Suppose that $\delta_{\Lambda}$ is tempered, and that $\widehat{\delta_{\Lambda}}$ is a Radon measure on $\mathbb{R}^{d}$ which satisfies

$$
\left|\widehat{\delta_{\Lambda}}\right|\left([-R, R]^{d}\right) \leq C R^{d}, \quad \text { as } \quad R \rightarrow \infty,
$$

where $C>0$ is a constant. Then, for each $s \in S$, the set

$$
\Lambda_{s}=\left\{\lambda \in \Lambda: c_{\lambda}=s\right\}
$$

is in the coset ring of $\mathbb{R}^{d}$.

A proof of Meyer's theorem for $d=1$ can be found in [KL]. The proof works verbatim for all $d$.

\subsection{Discrete Elements of the Coset Ring of a General Group}

In this section we determine the structure of the discrete elements of the coset ring of $\mathbb{R}^{d}$.

In dimension $d=1$ we have the following characterization of the discrete elements of the coset ring of $\mathbb{R}$, due to Rosenthal [R1].

Theorem (Rosenthal). The elements of the coset ring of $\mathbb{R}$ which are discrete in the usual topology of $\mathbb{R}$ are precisely the sets of the form

$$
F \triangle \bigcup_{j=1}^{J}\left(\alpha_{j} \mathbb{Z}+\beta_{j}\right),
$$

where $F \subseteq \mathbb{R}$ is finite, $\alpha_{j}>0$, and $\beta_{j} \in \mathbb{R}(\triangle$ denotes symmetric difference $)$.

Rosenthal's proof does not extend to dimension $d \geq 2$. Since we need to know what kind of sets the elements of the coset ring of $\mathbb{R}^{2}$ are, we prove the following general theorem, which says that discrete elements of the coset ring can always be constructed from discrete cosets using finitely many unions, intersections, and complementations.

Theorem 3. Let $G$ be a topological abelian group and let $\mathcal{R}$ be the least ring of sets which contains the discrete cosets of $G$. Then $\mathcal{R}$ contains all discrete elements of the coset ring of $G$. 
In other words, a discrete element of the coset ring can always be written as a finite union of sets of the type

$$
A_{1} \cap \cdots \cap A_{m} \cap B_{1}^{c} \cap \cdots \cap B_{n}^{c},
$$

where the $A_{i}$ and $B_{i}$ are discrete cosets of $G$. Observing that the intersection of any two cosets is a coset, we may rewrite (10) as

$$
A \cap B_{1}^{c} \cap \cdots \cap B_{n}^{c}
$$

where $A$ and all $B_{i}$ are discrete cosets.

We need the following lemma.

Lemma 2. Suppose that $A$ is a nondiscrete topological abelian group, $F \subset A$ is discrete, and $B_{1}, \ldots, B_{m}$ are cosets in A disjoint from $F$. Then

$$
A=F \cup B_{1} \cup \cdots \cup B_{n}
$$

implies that $F=\emptyset$. This remains true if $A$ is a nondiscrete coset in a larger group.

Proof of Lemma 2. Write $B_{i}=x_{i}+G_{i}$ and let $k$ be the number of different subgroups $G_{i}$ appearing in (12). We do induction on $k$. Notice that the group $G_{1}$ may be assumed to be nondiscrete, by the nondiscreteness of $A$.

When $k=1$ the theorem is true as then $F$ is a union of cosets of $G_{1}$ and cannot be discrete unless it is empty. (Here is where the disjointness of $F$ from the $B_{i}$ is used.)

Assume the theorem is true for $k \leq n$ and suppose that precisely $n+1$ groups appear in (12) and that $F \neq \emptyset$. Assume that the $G_{1}$-cosets in (12) are

$$
x_{1}+G_{1}, \ldots, x_{r}+G_{1},
$$

and let $y \in F$. We then have

$$
y+G_{1} \subseteq F \cup\left(X_{2}+G_{2}\right) \cup \cdots \cup\left(X_{n+1}+G_{n+1}\right),
$$

with all sets $X_{i}, i=2, \ldots, n+1$, being finite. Hence

$$
\begin{aligned}
G_{1} & \subseteq(-y+F) \cup\left(-y+X_{2}+G_{2}\right) \cup \cdots \cup\left(-y+X_{n+1}+G_{n+1}\right) \\
& =F^{\prime} \cup\left(X_{2}^{\prime}+G_{2}\right) \cup \cdots \cup\left(X_{n+1}^{\prime}+G_{n+1}\right),
\end{aligned}
$$

with $F^{\prime}=-y+F, X_{i}^{\prime}=-y+X_{i}$.

Furthermore, one may take $X_{i}^{\prime} \subset G_{1}, i=2, \ldots, n+1$ (possibly empty), to get

$$
G_{1}=\left(F^{\prime} \cap G_{1}\right) \cup\left(X_{2}^{\prime}+G_{2} \cap G_{1}\right) \cup \cdots \cup\left(X_{n+1}^{\prime}+G_{n+1} \cap G_{1}\right) .
$$

Since $y \in F$ we have that $F^{\prime} \cap G_{1} \ni 0$ (hence it is nonempty) and

$$
\left(F^{\prime} \cap G_{1}\right) \cap\left(X_{i}^{\prime}+G_{i} \cap G_{1}\right)=\emptyset, \quad i=2, \ldots, n+1 .
$$

By the induction hypothesis we get a contradiction. 
Proof of Theorem 3. By Lemma 2, if $A$ is nondiscrete, then $A \cap B_{1}^{c} \cap \cdots \cap B_{n}^{c}$ is either nondiscrete or empty. Hence a finite union of such sets can only be discrete if all participating $A$ 's are discrete. Rewrite then

$$
A \cap B_{1}^{c} \cap \cdots \cap B_{n}^{c}=A \cap\left(B_{1} \cap A\right)^{c} \cap \cdots \cap\left(B_{n} \cap A\right)^{c}
$$

so as to have the arbitrary discrete element of the coset ring made up with finitely many operations from discrete cosets.

\subsection{Discrete Elements of the Coset Ring of $\mathbb{R}^{2}$}

In this section we specialize the results of the previous section to the group $\mathbb{R}^{2}$.

Definition 6 (Dimension, Lattices). The dimension of a set $A \subseteq \mathbb{R}^{d}$ is the dimension of the smallest translated subspace of $\mathbb{R}^{d}$ that contains $A$. A lattice is a discrete subgroup of $\mathbb{R}^{d}$.

Remark. It is well known that all $k$-dimensional lattices in $\mathbb{R}^{d}$ are of the form $A \mathbb{Z}^{k}$, where $A$ is a $d \times k$ real matrix of rank $k$.

Theorem 4. Let $C=A \cap B_{1}^{c} \cap \cdots \cap B_{n}^{c}$, with $A, B_{i}$ being discrete cosets of $\mathbb{R}^{d}$. Then $C$ may be written as a finite (possibly empty) union of sets of the type

$$
K \cap L_{1}^{c} \cap \cdots \cap L_{m}^{c}, \quad L_{i} \subseteq K \subseteq A, \quad m \geq 1,
$$

where the $K, L_{i}$ are discrete cosets and, when $C$ is not empty,

$$
\operatorname{dim} L_{i}<\operatorname{dim} K=\operatorname{dim} A=\operatorname{dim} C \quad(i=1, \ldots, m) .
$$

Observation 2. If $A$ and $B$ are discrete cosets in $\mathbb{R}^{d}$ with $\operatorname{dim} A=\operatorname{dim} B=\operatorname{dim}(A \cap$ $B$ ), then $A \cap B^{c}$ is a finite (possibly empty) union of disjoint cosets of $A \cap B$ and, therefore, $\operatorname{dim}\left(A \cap B^{c}\right)=\operatorname{dim} A$, except when $A \cap B^{c}=\emptyset$. Hence $A$ and $B$ can each be written as a finite disjoint union of translates of $A \cap B$.

Proof of Theorem 4. Notice that

$$
C=A \cap\left(B_{1} \cap A\right)^{c} \cap \cdots \cap\left(B_{n} \cap A\right)^{c} .
$$

Let $r \geq 0$ be such that

$$
\alpha=\operatorname{dim} A=\operatorname{dim}\left(B_{1} \cap A\right)=\cdots=\operatorname{dim}\left(B_{r} \cap A\right)
$$

and $\operatorname{dim}\left(B_{i} \cap A\right)<\alpha$ for $i>r$. Let

$$
C^{\prime}=A \cap\left(B_{1} \cap A\right)^{c} \cap \cdots \cap\left(B_{r} \cap A\right)^{c} .
$$


By induction on $r \geq 0$ we prove that $C^{\prime}$ is a finite union of sets of type (13) satisfying (14). For $r=0$ this is obvious. If it is true for $r-1$, then $C^{\prime}$ is a finite union of sets of type

$$
K \cap L_{1}^{c} \cap \cdots \cap L_{m}^{c} \cap\left(B_{r} \cap A\right)^{c},
$$

with $\alpha=\operatorname{dim} K>\operatorname{dim} L_{i}, i=1, \ldots, m$. Each of these sets falls into one of two categories:

Category 1: $\operatorname{dim}\left(K \cap\left(B_{r} \cap A\right)\right)=\alpha$. Then, by Observation 2 above, $K \cap\left(B_{r} \cap A\right)^{c}$ is a finite union of cosets $K_{1}, \ldots, K_{s}$ of dimension $\alpha$ and hence $C^{\prime}$ is a finite union of $K_{i} \cap L_{1}^{c} \cap \cdots \cap L_{m}^{c}, i=1, \ldots, s$.

Category 2: $\operatorname{dim}\left(K \cap\left(B_{r} \cap A\right)\right)<\alpha$. Then

$$
K \cap L_{1}^{c} \cap \cdots \cap L_{m}^{c} \cap\left(B_{r} \cap A\right)^{c}
$$

is already of the desired form.

From Theorems 3 and 4 it follows for $d=2$ that every discrete element $S$ of the coset ring of $\mathbb{R}^{2}$ may be written in the form

$$
S=\left(\bigcup_{j=1}^{J} A_{j} \backslash\left(B_{1}^{(j)} \cup \cdots \cup B_{n_{j}}^{(j)}\right)\right) \cup \bigcup_{l=1}^{L} L_{l} \Delta F,
$$

where $A_{1}, \ldots, A_{J}$ are two-dimensional translated lattices, $L_{l}$ and $B_{i}^{(j)}$ are onedimensional translated lattices and $F$ is a finite set $(J, L \geq 0)$. Repeatedly using Observation 2, the lattices $A_{j}$ may be assumed to be have pairwise intersections of dimension at most one.

\subsection{Purely Discrete Fourier Transform}

Definition 7 (Uniform Density). A multiset $\Lambda \subseteq \mathbb{R}^{d}$ has asymptotic density $\rho$ if

$$
\lim _{R \rightarrow \infty} \frac{\#\left(\Lambda \cap B_{R}(x)\right)}{\left|B_{R}(x)\right|} \rightarrow \rho
$$

uniformly in $x \in \mathbb{R}^{d}$. We write $\rho=$ dens $\Lambda$.

We say that $\Lambda$ has (uniformly) bounded density if the fraction above is bounded by a constant $\rho$ uniformly for $x \in \mathbb{R}$ and $R>1$. We say then that $\Lambda$ has density (uniformly) bounded by $\rho$.

Assume that $\Lambda \subset \mathbb{R}^{2}$ is a discrete multiset of bounded density which satisfies the assumptions of Meyer's theorem (if we write $c_{\lambda}$ for the multiplicity of $\lambda \in \Lambda$ ). Then, if $\Lambda_{k}$ is the subset of $\Lambda$ of multiplicity $k, \Lambda_{k}$ is a discrete element of the coset ring and is of the form (15). 
Assume now in addition that $\widehat{\delta_{\Lambda}}$ has discrete support. We shall prove (Theorem 6 below) that all sets $F, L_{l}$, and $B_{i}^{(j)}$ are empty in (15) and so

$$
\Lambda=\bigcup_{j=1}^{J} A_{j},
$$

where the $A_{i}$ are translated two-dimensional lattices in $\mathbb{R}^{2}$, which intersect pairwise at most on dimension one.

One can easily show that whenever $\Omega \subseteq \mathbb{R}^{d}$ of finite measure tiles with $\Lambda$ at level $w$ then $\Lambda$ has density $w /|\Omega|$.

Theorem 5. Suppose that $\Lambda \in \mathbb{R}^{d}$ is a multiset with density $\rho, \delta_{\Lambda}=\sum_{\lambda \in \Lambda} \delta_{\lambda}$, and that $\widehat{\delta_{\Lambda}}$ is a measure in a neighborhood of zero. Then $\widehat{\delta_{\Lambda}}(\{0\})=\rho$.

Proof of Theorem 5. Take $\varphi \in C^{\infty}$ of compact support with $\varphi(0)=1$. We have

$$
\begin{aligned}
\widehat{\delta_{\Lambda}}(\{0\}) & =\lim _{t \rightarrow \infty} \widehat{\delta_{\Lambda}}(\varphi(t x)) \\
& =\lim _{t \rightarrow \infty} \delta_{\Lambda}\left(t^{-d} \widehat{\varphi}(\xi / t)\right) \\
& =\lim _{t \rightarrow \infty} t^{-d} \sum_{\lambda \in \Lambda} \widehat{\varphi}(\lambda / t) \\
& =\lim _{t \rightarrow \infty} \sum_{n \in \mathbb{Z}^{d}} \sum_{\lambda \in Q_{n}} t^{-d} \widehat{\varphi}(\lambda / t),
\end{aligned}
$$

where, for fixed and large $T>0$,

$$
Q_{n}=[0, T)^{d}+T n, \quad n \in \mathbb{Z}^{d} .
$$

Since $\Lambda$ has density $\rho$ it follows that for each $\varepsilon>0$ we can choose $T$ large enough so that, for all $n$,

$$
\left|\Lambda \cap Q_{n}\right|=\rho\left|Q_{n}\right|\left(1+\delta_{n}\right),
$$

with $\left|\delta_{n}\right| \leq \varepsilon$. For each $n$ and $\lambda \in Q_{n}$ we have

$$
\widehat{\varphi}(\lambda / t)=\widehat{\varphi}(T n / t)+r_{\lambda}
$$

with $\left|r_{\lambda}\right| \leq C T t^{-1}\|\nabla \widehat{\varphi}\|_{L^{\infty}\left(t^{-1} Q_{n}\right)}$. Hence

$$
\begin{aligned}
\widehat{\delta_{\Lambda}}(\{0\})= & \lim _{t \rightarrow \infty} \sum_{n \in \mathbb{Z}^{d}} t^{-d} \sum_{\lambda \in Q_{n}}\left(\widehat{\varphi}(T n / t)+r_{\lambda}\right) \\
= & \lim _{t \rightarrow \infty} \sum_{n \in \mathbb{Z}^{d}} t^{-d} \rho\left|Q_{n}\right|\left(1+\delta_{n}\right) \widehat{\varphi}(T n / t) \\
& +\lim _{t \rightarrow \infty} \sum_{n \in \mathbb{Z}^{d}} t^{-d} \sum_{\lambda \in Q_{n}} r_{\lambda} \\
= & \lim _{t \rightarrow \infty} S_{1}+\lim _{t \rightarrow \infty} S_{2} .
\end{aligned}
$$


We have

$$
\left|S_{1}-\sum_{n} t^{-d} \rho\right| Q_{n}|\widehat{\varphi}(T n / t)| \leq \varepsilon \sum_{n} t^{-d} \rho\left|Q_{n}\right||\widehat{\varphi}(T n / t)| .
$$

The first sum in (16) is a Riemann sum for $\rho \int_{\mathbb{R}^{d}} \widehat{\varphi}=\rho$ and the second is a Riemann sum for $\rho \int_{\mathbb{R}^{d}}|\widehat{\varphi}|<\infty$.

For $S_{2}$ we have

$$
\begin{aligned}
\left|S_{2}\right| & \leq C \sum_{n \in \mathbb{Z}^{d}} t^{-d} \rho\left|Q_{n}\right|\left(1+\delta_{n}\right) T t^{-1}\|\nabla \widehat{\varphi}\|_{L^{\infty}\left(t^{-1} Q_{n}\right)} \\
& \leq C \rho T t^{-1} \sum_{n \in \mathbb{Z}^{d}} t^{-d}\left|Q_{n}\right|\|\nabla \widehat{\varphi}\|_{L^{\infty}\left(t^{-1} Q_{n}\right)} .
\end{aligned}
$$

The sum above is a Riemann sum for $\int_{\mathbb{R}^{d}}|\nabla \widehat{\varphi}|$, which is finite, hence $\lim _{t \rightarrow \infty} S_{2}=0$.

Since $\varepsilon$ is arbitrary the proof is complete.

Remark. The same proof as that of Theorem 5 shows that if

$$
\mu=\sum_{\lambda \in \Lambda} c_{\lambda} \delta_{\lambda}
$$

with $\left|c_{\lambda}\right| \leq C, \Lambda$ is of density zero, and the tempered distribution $\widehat{\mu}$ is locally a measure in the neighborhood of some point $a \in \mathbb{R}^{2}$, then we have $\widehat{\mu}(\{a\})=0$.

Theorem 6. Suppose that $\Lambda \subset \mathbb{R}^{2}$ is a discrete multiset of uniformly bounded density and that

$$
\widehat{\delta_{\Lambda}}=\left(\sum_{\lambda \in \Lambda} \delta_{\lambda}\right)^{\wedge}
$$

is locally a measure with

$$
\left|\widehat{\delta_{\Lambda}}\right|\left(B_{R}(0)\right) \leq C R^{2},
$$

for some positive constant $C$ and $R \geq 1$. Assume also that $\widehat{\delta_{\Lambda}}$ has discrete support. Then $\Lambda$ is a finite union of translated lattices.

Proof of Theorem 6. Define the sets (not multisets)

$$
\Lambda_{k}=\{\lambda \in \Lambda: \lambda \text { has multiplicity } k\} .
$$

By Meyer's theorem (applied for the base set of the multiset $\Lambda$ with the coefficients $c_{\lambda}$ equal to the corresponding multiplicities) each of the $\Lambda_{k}$ is in the coset ring of $\mathbb{R}^{2}$ and, being discrete, is of the type (15).

We may thus write

$$
\Lambda_{k}=A \triangle B,
$$

with $A=\bigcup_{j=1}^{J} A_{j}$, where the two-dimensional translated lattices $A_{j}$ have pairwise intersections of dimension at most one, and dens $B=0$. 
Hence

$$
\delta_{\Lambda_{k}}=\sum_{j=1}^{J} \delta_{A_{j}}+\mu,
$$

where $\mu=\sum_{f \in F} c_{f} \delta_{f}$, dens $F=0$, and $\left|c_{f}\right| \leq C(J)$. The set $F$ consists of $B$ and all points contained in at least two of the $A_{j}$.

Combining for all $k$, and reusing the symbols $A_{j}, \mu$, and $F$, we get

$$
\delta_{\Lambda}=\sum_{j=1}^{J} \delta_{A_{j}}+\mu .
$$

However, $\widehat{\delta_{\Lambda}}$ and $\sum_{j=1}^{J} \widehat{\delta_{A_{j}}}$ are both (by the assumption and the Poisson Summation Formula) discrete measures, and so is therefore $\widehat{\mu}$. However, dens $F=0$ and the boundedness of the coefficients $c_{f}$ implies that $\widehat{\mu}$ has no point masses (see the Remark after the proof of Theorem 5), which means that $\widehat{\mu}=0$ and so is $\mu$. Hence $\delta_{\Lambda}=\sum_{j=1}^{J} \delta_{A_{j}}$, or

$$
\Lambda=\bigcup_{j=1}^{J} A_{j}, \quad \text { as multisets. }
$$

Finally, we show that discrete support for $\widehat{\delta_{\Lambda}}$ implies that $\widehat{\delta_{\Lambda}}$ is locally a measure.

Theorem 7. Suppose that the multiset $\Lambda \subset \mathbb{R}^{d}$ has density uniformly bounded by $\rho$ and that, for some point $a \in \mathbb{R}^{d}$ and $R>0$,

$$
\operatorname{supp} \widehat{\delta_{\Lambda}} \cap B_{R}(a)=\{a\} .
$$

Then, in $B_{R}(a)$, we have $\widehat{\delta_{\Lambda}}=w \delta_{a}$, for some $w \in \mathbb{C}$ with $|w| \leq \rho$.

Proof of Theorem 7. It is well known that the only tempered distributions supported at a point $a$ are finite linear combinations of the derivatives of $\delta_{a}$. So we may assume that, for $\varphi \in C^{\infty}\left(B_{R}(a)\right)$,

$$
\widehat{\delta_{\Lambda}}(\varphi)=\sum_{\alpha} c_{\alpha}\left(D^{\alpha} \delta_{a}\right)(\varphi)=\sum_{\alpha}(-1)^{|\alpha|} c_{\alpha} D^{\alpha} \varphi(a)
$$

where the sum extends over all values of the multi-index $\alpha=\left(\alpha_{1}, \ldots, \alpha_{d}\right)$ with $|\alpha|=$ $\alpha_{1}+\cdots+\alpha_{d} \leq m$ (the finite degree) and $D^{\alpha}=\partial_{1}^{\alpha_{1}} \cdots \partial_{d}^{\alpha_{d}}$ as usual.

We want to show that $m=0$. Assume the contrary and let $\alpha_{0}$ be a multi-index that appears in (18) with a nonzero coefficient and has $\left|\alpha_{0}\right|=m$. Pick a smooth function $\varphi$ supported in a neighborhood of zero which is such that for each multi-index $\alpha$ with $|\alpha| \leq m$ we have $D^{\alpha} \varphi(0)=0$ if $\alpha \neq \alpha_{0}$ and $D^{\alpha_{0}} \varphi(0)=1$. (To construct such a $\varphi$, multiply the polynomial $\left(1 / \alpha_{0} !\right) x^{\alpha_{0}}$ with a smooth function supported in a neighborhood of zero, which is identically equal to one in a neighborhood of zero.)

For $t \rightarrow \infty$ let $\varphi_{t}(x)=\varphi(t(x-a))$. Equation (18) then gives that

$$
\widehat{\delta_{\Lambda}}\left(\varphi_{t}\right)=t^{m}(-1)^{m} c_{\alpha_{0}} .
$$


On the other hand, using

$$
(\varphi(t(x-a)))^{\wedge}(\xi)=e^{-2 \pi i\langle a, \xi / t\rangle} t^{-d} \widehat{\varphi}(\xi / t),
$$

we get

$$
\widehat{\delta_{\Lambda}}\left(\varphi_{t}\right)=\sum_{\lambda \in \Lambda} e^{-2 \pi i\langle a, \lambda / t\rangle} t^{-d} \widehat{\varphi}(\lambda / t)
$$

Notice that (20) is a bounded quantity as $t \rightarrow \infty$ by a proof similar to that of Theorem 5 , while (19) increases like $t^{m}$, a contradiction.

Hence $\widehat{\delta_{\Lambda}}=w \delta_{a}$ in a neighborhood of $a$. The proof of Theorem 5 again gives that $|w| \leq \rho$.

Using Theorem 7 we may drop from Theorem 6 the assumption that $\widehat{\delta_{\Lambda}}$ has to be locally a measure, as this is now implied by the discrete support which we assume for $\widehat{\delta_{\Lambda}}$. Summing up we have the following.

Theorem 8. Suppose that the multiset $\Lambda$ has uniformly bounded density, that $S=$ $\operatorname{supp} \widehat{\delta_{\Lambda}}$ is discrete, and that

$$
\left|S \cap B_{R}(0)\right| \leq C R^{d},
$$

for some positive constant $C$. Then $\Lambda$ is a finite union of translated d-dimensional lattices.

\subsection{Application to Tilings by Polygons}

In this section we apply Theorem 8 and the characterization of the zero-sets of the functions $\widehat{\mu_{e, \tau}}$ (Theorem 1) in order to give very general sufficient conditions for a polygon $K$ to admit only quasi-periodic tilings, if it tiles at all.

Theorem 9. Let the polygon $K$ have the pairing property and let it tile multiply the plane with the multiset $\Lambda$. Denote the edges of $K$ by (we follow the notation of Section 1.1)

$$
e_{1}, e_{1}+\tau_{1}, e_{2}, e_{2}+\tau_{2}, \ldots, e_{n}, e_{n}+\tau_{n} .
$$

Suppose also that

$$
\left\{\widetilde{e_{1}}, \widetilde{\tau_{1}}\right\} \cap \cdots \cap\left\{\widetilde{e_{n}}, \widetilde{\tau_{n}}\right\}=\emptyset,
$$

where with $\widetilde{v}$ we denote the orientation of vector $v^{\perp}$. Then $\Lambda$ is a finite union of translated two-dimensional lattices.

Proof of Theorem 9. By Theorem 1 and the tiling assumption we get

$$
\operatorname{supp} \widehat{\delta_{\Lambda}} \subseteq Z\left(\widehat{\mu_{e_{1}, \tau_{1}}}\right) \cap \cdots \cap Z\left(\widehat{\mu_{e_{n}, \tau_{n}}}\right)
$$

By Theorem 1 in the intersection above each of the sets is contained in a collection of lines in the direction $\widetilde{e}_{i}$ union a collection of lines in the direction $\widetilde{\tau}_{i}$. Because of assumption (21) these sets have a discrete intersection as two lines of different orientations 
intersect at a point. Furthermore, because of the regular spacing of these pairs of sets of lines, it follows that the resulting intersection has at most $C R^{2}$ points in a large disk of radius $R$. Theorem 8 now implies that $\Lambda$ is a finite union of translated two-dimensional lattices.

Condition (21) is particularly easy to check for convex polygons.

Theorem 10. Suppose that $K$ is a symmetric convex polygon which is not a parallelogram. Then $K$ admits only quasi-periodic multiple tilings.

Proof of Theorem 10. Suppose that (21) fails and that the intersection in (21) contains a vector which is, say, parallel to the $y$-axis. It follows that each pair of edges $e_{i}, e_{i}+\tau_{i}$ of edges of $K$ either (a) has both edges parallel to the $x$-axis, or (b) has the line joining the two midpoints parallel to the $x$-axis. As this latter line goes through the origin it is clear that (b) can only happen for one pair of edges and, since (a) cannot happen for two consecutive pairs of edges, (a) can hold at most once as well. This means that $K$ is a parallelogram.

Remarks. 1. It is clear that parallelograms admit tilings which are not quasi-periodic. Take for example the regular tiling by a square and move each vertical column of squares arbitrarily up or down.

2. Some very intersecting classes of polygons are left out of reach of Theorem 9. An important class consists of all polygons whose edges are parallel to either the $x$ - or the $y$-axis.

\section{References}

[B] U. Bolle, On multiple tiles in $E^{2}$, in Intuitive Geometry, K. Böröczky and G. Fejes Tóth, editors, Colloq. Math. Soc. J. Bolyai 63, North-Holland, Amsterdam, 1994.

[GS] B. Grünbaum and G. C. Shephard, Tilings and Patterns, Freeman, New York, 1987.

[K1] Y. Katznelson, An Introduction to Harmonic Analysis, Wiley, New York, 1968, and Dover, New York, 1976.

[K2] M. N. Kolountzakis, Lattice tilings by cubes: whole, notched and extended, Electron. J. Combin. 5 (1998), 1, R14.

[KL] M. N. Kolountzakis and J. C. Lagarias, Structure of tilings of the line by a function, Duke Math. J. 82(3), (1996), 3, 653-678.

[M] Y. Meyer, Nombres de Pisot, nombres de Salem et analyse harmonique, Lect. Notes Math. 117, SpringerVerlag, Berlin, 1970.

[R1] H. P. Rosenthal, Projections onto Translation-Invariant Subspaces of $L^{P}(G)$, Mem. Amer. Math. Soc. 63, American Mathematical Society, Providence, RI, 1966.

[R2] W. Rudin, Functional Analysis, McGraw-Hill, New York, 1973.

[SS] S. K. Stein and S. Szabó, Algebra and Tiling. Homomorphisms in the Service of Geometry, Mathematical Association of America, Washington, DC, 1994.

Received February 24, 1999, and in revised form August 26, 1999, and October 9, 1999. 